\title{
Tamouls du Sri Lanka en France : politisation de l'exil dans l'ombre des Tigres
}

Tamil of Sri Lanka in France: Politization of the Exile in the Shade of the Tigers

Tamiles del Sri Lanka en Francia: politización del exilio en la sombra de los

Tigres

\section{Angélina Étiemble}

\section{(2) OpenEdition}

Journals

\section{Édition électronique}

URL : https://journals.openedition.org/remi/9376

DOI : $10.4000 /$ remi.9376

ISSN : $1777-5418$

Éditeur

Université de Poitiers

Édition imprimée

Date de publication : 1 décembre 2017

Pagination : 65-86

ISBN : 979-10-90426-60-3

ISSN : 0765-0752

Référence électronique

Angélina Étiemble, «Tamouls du Sri Lanka en France : politisation de l'exil dans l'ombre des Tigres », Revue européenne des migrations internationales [En ligne], vol. 33 - n4 | 2017, mis en ligne le 01 décembre 2019, consulté le 14 avril 2022. URL : http://journals.openedition.org/remi/9376 ; DOI : https://doi.org/10.4000/remi.9376 


\section{Tamouls du Sri Lanka en France: politisation de l'exil dans l'ombre des Tigres Angélina Étiemble ${ }^{1}$}

Au Sri Lanka, I'un des plus longs conflits armés contemporains prend fin au mois de mai 2009. II opposait depuis 1983 les forces gouvernementales, liées à la majorité cinghalaise, et les séparatistes des Tigres de la Libération du Tamil Eelam (LTTE), organisation paramilitaire autoproclamée représentante de la minorité tamoule. Ce conflit, violent, s'achève par la défaite des séparatistes, symbolisée par la mort de leur leader, Velupillai Prabhakaran. II a conduit des milliers de Tamouls à chercher refuge loin de leur île, notamment en Europe. Dans les années 1980, la France n'est pas la destination d'élection des premiers exilésTamouls, a contrario de l'Angleterre. Elle le devient au fil du temps puisque les Sri-Lankais, et parmi eux surtout des Tamouls, y représentent la première population réfugiée statutaire encore aujourd'hui. L'installation " communautaire " des Tamouls, visible dans la région parisienne, et le traitement favorable de leur demande d'asile dans la décennie 1990 expliquent en grande partie cette configuration. Plus encore, les sympathisants des LTTE, très présents dans le quotidien des réfugiés et de leurs enfants, encouragent leur "intégration ${ }^{2}$ en France en définissant ses contours : soutenir la " cause tamoule ». Leur influence se fait particulièrement ressentir à partir de 1995 avec la création du Comité de Coordination des Tamouls (CCT) en France. Le CCT est une émanation directe de I'organisation des LTTE, plus communément nommée le Mouvement. Ses "filiales" associatives vont contribuer à l'encadrement idéologique des réfugiés et de leurs enfants. Chez nombre d'entre eux se renforce une lecture nationaliste de l'exil. C'est dans cette période (1998-2001) et ce contexte que nous réalisons

\footnotetext{
1 Sociologue, maîtresse de conférences, VIPS², Le Mans Université, UFR Sciences, avenue Olivier Messiaen, 72085 Le Mans cedex 9 ; angelina.etiemble@univ-lemans.fr 2 Les expressions en italiques sont celles des enquêtés.
} 
une trentaine d'entretiens ${ }^{3}$ avec des Tamouls habitant la région parisienne et engageons des observations "flottantes" (Pétonnet, 1982) dans le secteur de La Chapelle, au gré des lieux identifiés comme ceux d'une présence physique et symbolique des Tamouls : temples, locaux de traducteurs, commerces, écoles tamoules, Comité de Coordination des Tamouls, mais aussi centre social de quartier et associations telles le Secours catholique. Nous menons une première investigation en lien avec un programme du FAS (Fonds d'action sociale) consacré aux "Nouvelles immigrations " et la poursuivons dans le cadre du programme de la MiRe/Drees (la Mission Recherche de la Direction de la recherche, des études, de l'évaluation et des statistiques) relatif aux "Circulations migratoires ". Ce programme (1999) vise à interroger l'intensification et la diversification des flux migratoires, ainsi que la variété des expériences selon les migrants et les pays, en croisant les disciplines (notamment géographie, sociologie et économie) et les outils d'investigation, approches statistiques (exploitation de données européennes et nationales) et enquêtes qualitatives de terrain (Costa-Lascoux et Du Cheyron, 2004). C'est dans I'un des thèmes du programme, relatif aux " facteurs et modalités des circulations transfrontalières ", que nous nous inscrivons avec pour ambition d'étudier le mouvement de Tamouls réfugiés en France vers l'Angleterre. En effet, précédemment, nous nous sommes intéressées aux trajectoires professionnelles, familiales et résidentielles des réfugiés rencontrés (Étiemble, 2000). Ces trajectoires sont très marquées par la présence, de la "communauté tamoule " sri-lankaise tant pour I'accès à l'emploi, la recherche ou l'acquisition d'un logement que pour l'éducation des enfants et la vie culturelle. Par " communauté ", nos interlocuteurs désignent les Tamouls du Sri Lanka installés dans la région parisienne et se reconnaissant dans un mode de vie, une histoire et des centres d'intérêt proches. L'entre-soi y paraît développé et l'espace, physique et symbolique, fortement occupé par la présence des sympathisants des LTTE. Pour autant, nous avons I'occasion d'entendre desTamouls formuler le vœu de quitter la France pour l'Angleterre et, d'une certaine façon, rompre avec cette " communauté " très ancrée sur le sol parisien. Nous envisageons alors d'étudier ce projet de traverser les frontières, dans l'hypothèse d'une perspective "transnationale " (Portes, 1999). Cependant, nous nous heurtons rapidement à la difficulté de retrouver ceux qui auraient entrepris cette migration et, surtout, constatons les entraves à cette circulation, les réfugiés tamouls étant en quelque sorte arrimés en France par l'antienne des LTTE concernant le sens de l'exil et de la migration. Nous proposons ici de mieux comprendre cette entrave, qui nous paraît significative

\footnotetext{
3 Nous mettons en œuvre la " méthode boule-de-neige " pour décrocher des entretiens avec des personnes déjà familières de la langue française ou tout au moins anglophones. Cette méthode a pour avantage de nous orienter d'emblée vers des personnes suffisamment en confiance pour accepter un entretien et, pour désavantage, de cibler un réseau d'interconnaissance. Nous allons donc également faire du porte-à-porte, par exemple, dans les officines de traducteurs assermentés - souvent originaires de Pondichéry - leur expliquant notre intérêt pour la vie des Tamouls du Sri Lanka en France et les sollicitant pour contacter, avec leur accord, leurs clients. Nous aurons ainsi l'occasion de côtoyer d'autres Tamouls, dont certains se montrant plus distants à l'égard des LTTE. Les personnes rencontrées connaissent en général des situations professionnelles et/ou économiques assez précaires, même si un nombre non négligeable est propriétaire de son logement. Elles sont inscrites dans des secteurs d'activité tels que le commerce (épicerie, restauration), le gardiennage et la sécurité, le service en restauration, I'aide à domicile, le ménage, la formation, la comptabilité, le secrétariat, etc. La plupart sont mariées et parents de jeunes enfants ou d'adolescents ; arrivées en France parfois depuis plus de vingt ans, d'autres plus récemment ou, pour les plus jeunes, nées en France.
} 
de l'emprise politique exercée par les militants nationalistes sur les réfugiés tamouls dans les années précédant la fin du conflit au Sri Lanka.

Après avoir rappelé la genèse de l'exil, s'enracinant dans le conflit opposant la majorité cinghalaise et la minorité tamoule, nous évoquerons les circonstances qui ont présidé, et renforcé, l'installation des exilés Tamouls en France, avec l'assentiment des militants nationalistes, distillant leur vision de l'exil et de "l'intégration " et invalidant tout projet de nouvelle migration. Nous nous attarderons ensuite sur le fonctionnement et le rôle du secteur associatif dans la politisation des réfugiés tamouls, tout au moins jusqu'à l'interdiction de l'organisation des LTTE en 2006.

\section{Vivre en France au nom de la " cause tamoule "}

Lîle de Ceylan a connu plusieurs périodes de colonisation à partir du XVle siècle : les Portugais, les Hollandais, les Allemands, enfin, les Britanniques dès 1815 jusqu'en 1948, date de l'Indépendance. Ces derniers ont, davantage que les précédents, bouleversé les structures sociales et les barrières traditionnelles des castes et des religions en unifiant l'île sous un seul gouvernement et une administration centralisée. Ils ont ainsi généré de nouvelles tensions et conflits entre castes et entre les Cinghalais, groupe ethnique majoritaire, principalement bouddhiste, et les Tamouls, groupe ethnique minoritaire, principalement hindouiste, habitant le Nord du pays (la péninsule de Jaffna), l'Est et la capitale Colombo. Après I'Indépendance, la situation se dégrade avec l'arrivée au pouvoir de la majorité cinghalaise aux élections de 1956. Le gouvernement prend, en effet, différentes mesures pour " recinghaliser " l'île. La langue cinghalaise est promue la seule langue officielle et le bouddhisme, religion d'État. Le gouvernement favorise également l'implantation de paysans cinghalais sans terre dans des régions considérées par les Tamouls comme leur patrie. Au début des années 1970, des mesures dites de "standardisation " limitent l'entrée des Tamouls à I'Université et beaucoup y voient la mise en péril de leur identité et de leur condition sociale. Des groupes politiques tamouls contestent ces mesures discriminatoires, sans s'entendre toutefois sur les modalités d'action - violence ou non-violence - et restent divisés par les distinctions de caste. Ainsi, les membres du People's Liberation ofTamil Eelam (PLOTE) viennent largement de la caste dominante des Vellalar (les " agriculteurs " ou propriétaires terriens) tandis que les leaders des Tigres, de la basse caste des Karaiyar (les " pêcheurs "). Deux événements majeurs, I'incendie criminel de la grande bibliothèque tamoule à Jaffna en 1981 et les violentes émeutes anti-tamoules à Colombo en juillet $1983^{4}$, dans I'indifférence du gouvernement cinghalais, radicalisent les tensions. Les Tigres de la libération de l'Eelam tamoul entrent en guerre contre l'armée en 1983 et revendiquent l'indépendance du nord-est de l'île. Dans le même temps, ils réduisent au silence les autres groupes politiques tamouls.

\footnotetext{
42000 à 3000 personnes sont tuées en une semaine, brûlées vives le plus souvent. Les Tamouls parlent d'un véritable pogrom et le commémorent annuellement. II semblerait qu'une rumeur ait circulé dans la presse lors des semaines précédentes selon laquelle lesTigres allaient attaquer le temple de la Dent de Bouddha à Kandy, empoisonner I'eau et arriver dans le Sud à bord de trains en provenance de Jaffna. Rumeur attisant la haine et la peur de la population cinghalaise.
} 
Nombre de Sri-Lankais vont fuir la guerre et ses multiples violences : les attentats, les représailles militaires ainsi que l'enrôlement forcé dans les deux camps. Pendant près d'un quart de siècle, le Sri Lanka ${ }^{5}$, et notamment la région nord-est, exsangue, connaît une forte émigration. En 2000, le gouvernement estime à au moins 1 million le nombre de Sri-Lankais émigrés dans le monde, pour environ 19 millions d'habitants (Meyer, 2001). Avant même le conflit, le phénomène migratoire est déjà très ancré dans l'île. II a été impulsé par les Britanniques au XIXe siècle, qui encouragent les mobilités dans I'empire. II s'accentue au cours du XXe siècle en raison des opportunités d'emploi offertes par la manne pétrolière au Moyen-Orient, puis la situation de violence dans le pays affecte une population déjà très mobile. Au cours de la décennie 1990, selon le HCR, 200000 Tamouls seraient ainsi partis pour le Canada et l'Europe (HCR, 2000).

En Europe, la France est devenue une terre d'asile pour les Tamouls du Sri Lanka, quand bien même les premiers Sri-Lankais exilés cherchaient plutôt, via la France, à gagner l'Angleterre en raison des liens culturels avec I'ancienne puissance coloniale et l'installation de longue date de compatriotes, voire de parents. Mais le pays est devenu moins accessible après la fermeture de ses frontières aux citoyens du Commonwealth, excepté des catégories précises (chercheurs, étudiants, personnel médical, etc.). Le passage est donc difficile et nombre de Sri-Lankais sont contraints de rester en France et d'y solliciter leur protection au titre de l'asile.

\section{Se réfugier en France}

Les premiers arrivés, isolés, obtiennent rarement le statut de réfugié. Ils bénéficieront des opérations de régularisation des années 1980. Ils travaillent dans la restauration, le ménage ou la distribution de prospectus (Robuchon, 1995). Ils habitent des logements exigus et souvent insalubres des 10e et $18 \mathrm{e}$ arrondissements parisiens. Cette présence tamoule du Sri Lanka, assez inaperçue dans ces premières années, devient plus visible la décennie suivante. En effet, la communauté internationale a pris la mesure de la violence du conflit armé et ses conséquences dramatiques pour la minorité tamoule. La demande d'asile des ressortissants sri-lankais progresse fortement en Europe de l'Ouest, notamment en France où elle est la plus importante - $18 \%$ de l'ensemble des demandes d'asile sri-lankaises européennes. La demande d'asile sri-lankaise augmente régulièrement en France pour osciller entre 1800 et 2000 dossiers annuels à la fin de la décennie et au début des années 2000. Les dossiers font référence à l'insécurité et à la précarité générale au Sri Lanka, à la brutalité des autorités, aux arrestations, rafles et détentions à Colombo, enfin, aux menaces et exactions du LTTE $^{6}$. La population sri-lankaise est pendant plusieurs années I'une des premières à solliciter l'asile en France et à se voir reconnaître le statut de réfugié. Le nombre de réfugiés statutaires originaires du Sri Lanka place d'ailleurs la France au deuxième rang après I'Inde, respectivement comptant 15600 et 66400 réfugiés au 31 décembre 1999 (HCR, 2000). Encore, en 2001,

5 Ceylan devient Sri Lanka en 1972.

6 OFPRA, Rapport d'activité 2001, 52 p., [en ligne]. URL : https://ofpra.gouv.fr/sites/ default/files/atoms/files/rapport_dactivite_2001.pdf 
le taux d'admission global ${ }^{7}$ est de $43,9 \%$ pour les Sri-Lankais alors qu'il n'est que de $18 \%$ pour l'ensemble des nationalités. La fin du conflit en 2009 ne se traduit pas aussitôt par une baisse de la demande d'asile sri-lankaise en France. Au contraire, cette demande connait un pic en 2009 avec 3383 dossiers déposés à I'OFPRA, soit $33 \%$ de plus que l'année précédente. C'est seulement à partir de 2011 qu'elle commence à s'infléchir et à se transformer, les demandes de réexamen ${ }^{8}$ devenant avec le temps plus nombreuses que les premières demandes, et la reconnaissance du statut moins "évidente " que dans les années précédentes ${ }^{9}$. De 1993 à 2012, les Sri-Lankais représentent la première nationalité des personnes admises sous protection OFPRA. Au nombre de 23225 réfugiés statutaires (13,4\% de l'ensemble des réfugiés sous protection), en 2012, ils devancent les réfugiés du Cambodge, de RDC, de la Russie et de Turquie. Les Sri-Lankais comptent également parmi les nationalités traditionnellement les plus représentées chez les réfugiés qui renoncent au statut protecteur.

Ces chiffres démontrent combien les Tamouls du Sri Lanka ont élu la France comme terre d'asile et d'installation, pourtant cette réalité paraît peu connue, sinon visible au-delà de leur ancrage très territorialisé dans la région parisienne, et plus encore dans le quartier de la Chapelle à Paris. Ce quartier, situé entre la station de métro éponyme et la gare du Nord, à la lisière des 10e et 18 e arrondissements, est particulièrement prisé par les Tamouls du Sri Lanka. L'essaimage de commerces, temples hindouistes, associations et médias à destination d'un public tamoul lui donne une coloration singulière. Les Tamouls eux-mêmes I'appellent "Little Jaffna ", du nom de la capitale tamoule du Nord de l'île. En 2012, les Sri-Lankais sous protection résident principalement dans les départements Nord-Est de la région parisienne, notamment la Seine-SaintDenis, ainsi que dans I'Est de la France. Les données de I'INSEE rejoignent celles de I'OFPRA puisqu'en 2014 sont recensés 46653 immigrés ${ }^{10}$ sri-lankais, dont près de $87 \%$ résidant dans la région Île-de-France. Cette présence francilienne témoigne de la manière dont les Sri-Lankais se sont installés en France, en restant très attachés à la capitale et aux villes avoisinantes du Nord-Est, dans le prolongement du quartier parisien de La Chapelle ${ }^{11}$. L'impossibilité d'établir des statistiques ethniques dans toute opération de recensement ne nous permet pas

7 Admission au statut par I'OFPRA et la CRR.

8 Après le rejet de la demande d'asile par l'OFPRA, les personnes ont la possibilité de faire une nouvelle demande d'asile à condition d'apporter un "élément nouveau ". Si la demande est jugée recevable par I'OFPRA, elle fera l'objet d'un traitement accéléré.

9 En 2016, I'OFPRA enregistre 2274 demandes sri-lankaises, dont 1082 premières demandes et 1192 demandes de réexamen, observant néanmoins un retrait des premières demandes (59\% de baisse par rapport à 2009) et une augmentation des demandes de réexamen (OFPRA, Rapport d'activité 2016, 72 p., [en ligne]. URL : https:// ofpra.gouv.fr/sites/default/files/atoms/files/rapport_dactivite_ofpra_2016_1.pdf). Ces chiffres, qui ne concernent pas directement la période étudiée ici, laissent à penser que la situation au Sri Lanka est loin d'être politiquement apaisée, malgré la fin de la guerre.

10 Selon I'Insee, est immigrée toute personne née étrangère à l'étranger et résidant en France. Elle peut être devenue Française. C'est pourquoi les chiffres de l'immigration et de la population étrangère sont différents.

11 Le secteur de La Chapelle est traditionnellement un lieu d'accueil des immigrations récentes ; il paraît avoir également fonctionné comme un sas pour les premiers Tamouls du Sri Lanka, avant leur installation dans les quartiers proches ou dans les villes de la Petite Couronne desservies par les lignes du métro ou de la Grande Couronne par le réseau ferroviaire vers le Nord-Est, confortant la configuration communautaire. 
de différencier des "Tamouls " et des "Cinghalais " au sein de la population srilankaise. Cependant, nous supposons que de nombreuses personnes pourraient se déclarer tamoules, tant la demande d'asile des "Tamouls du Sri Lanka " a été élevée tout au long de la décennie 1990 et jusqu'à la fin de la guerre en 2009. Cette présence très localisée en France témoigne également d'une installation communautaire, dans l'ombre des sympathisants des LTTE. Ces derniers semblent, en effet, maintenir dans leur sillage politique les réfugiés tamouls à tel point qu'il parait difficile d'exprimer un propos quelque peu différent ou de faire advenir un projet de nouvelle migration, se distanciant de la lecture nationaliste de la migration.

\section{La mobilité migratoire stigmatisée par les militants nationalistes}

Nous l'avons dit, certains Tamouls, réfugiés statutaires en France, voire naturalisés Français, envisagent de s'installer en Angleterre. La précarité du séjour ne semble donc pas expliquer leur projet d'une nouvelle migration. Ils sont souvent présents en France depuis une dizaine d'années et parents d'enfants encore jeunes. Anglophones, ils estiment que leur connaissance de la langue anglaise leur facilitera l'accès à un emploi comparable à celui occupé avant l'exil. D'autres, moins familiers de la langue anglaise, suivent des cours, pour migrer en Angleterre et, selon eux, s'y " intégrer " alors plus facilement. Nos interlocuteurs motivent leur projet par les possibilités d'accéder, selon leurs termes, à un "bon travail ", à de "grandes maisons " et, enfin, être l'objet de "respect", autant d'éléments culturellement valorisés par les Tamouls et qu'ils ne pourraient connaître en France (Étiemble, 2002 : 176-190). Ils évoquent à I'envi des proches, "avocats" ou " médecins ", vivant en Angleterre de longue date, parfois depuis les années 1960. Pour les plus anciens, leur familiarité avec le système éducatif anglais serait la garantie de la réussite scolaire de leurs enfants, tant en Angleterre qu'au Sri Lanka, la langue et les diplômes anglais ayant là aussi plus grande valeur. Ils se réfèrent à la société tamoule d'avant la guerre, quand I'anglais était la langue usuelle de l'élite tamoule de Jaffna, influencée par la colonisation britannique. L'anglais est, en effet, l'apanage des personnes "éduquées", euphémisme tamoul pour désigner les membres de la caste dominante des Vellalar. Par ricochet, elle procure du prestige aux membres anglophones de basses castes. Grâce à leur instruction, ils deviennent à leur tour des personnes "éduquées ", s'émancipant de la hiérarchie des castes. Le projet d'installation en Angleterre paraît ainsi découler, dans les castes dominantes, du désir de retrouver une position sociale antérieure à l'exil et, dans les basses castes, de celui de l'ascension sociale. Nos interlocuteurs formulent plus rarement l'idée de s'éloigner des Tigres, de leurs menaces et de leurs collectes de fonds (cf. infra). D'autres auraient quitté la France pour échapper à l'opprobre familial après avoir fait un " mariage mixte ", entendu comme une union intercastes. Les enquêtés désireux de quitter la France pour l'Angleterre, comptent sur leur réseau familial et I'acquisition de la nationalité française pour réaliser leur projet. La naturalisation française est effectivement rapidement envisagée par les Tamouls après l'obtention du statut de réfugié ou la régularisation du séjour. Rappelons que les Sri-Lankais sont l'une des premières nationalités à renoncer à la protection de I'OFPRA. En 1999, ils sont d'ailleurs au douzième rang des personnes d'origine étrangère ayant acquis la nationalité française, au vingt-quatrième quatre ans plus tôt (Lebon, 2000). L'obtention de la nationalité 
est perçue comme une garantie de meilleure intégration, mais aussi facilitant les déplacements d'un pays à l'autre, rendre visite à la famille restée au pays ou dans d'autres pays d'installation des Sri-Lankais, voire pour y vivre à leur tour. Ces aspirations à une nouvelle migration afin de "vivre bien " semblent néanmoins peu se concrétiser tant de tels départs sont faibles. Nous n'en avons repéré qu'une dizaine lors de nos enquêtes. Le défaut d'opportunités se conjugue alors à la crainte de déplaire aux militants des LTTE et freine les mobilités migratoires et sociales.

Les militants du CCT, eux, ne se montrent pas hostiles à I'acquisition de la nationalité française, au contraire, dans la mesure où l'accès à la citoyenneté française donne, en quelque sorte, une arme électorale. À leurs yeux, Les Tamouls, devenus citoyens français, peuvent contribuer par leur vote, voire leur mandat politique, à défendre et légitimer la "cause tamoule " sur la scène politique française, jugée trop conciliante avec la présidence cinghalaise. La naturalisation n'est pas interprétée comme un renoncement à l'identité tamoule, mais plutôt comme un procédé pour la sauvegarder et la valoriser en France. De même, les " amis des LTTE ", tels qu'ils s'auto-désignent, désapprouvent les projets de départ vers l'Angleterre et encouragent, au contraire, "I'intégration " en France. Selon eux, toutefois, la "bonne intégration " se limite à la sphère socio-économique. Elle passe par l'apprentissage du français, une scolarisation ou une formation réussie aboutissant à un emploi qualifié. A contrario, les membres du CCT, et en particulier les militantes, s'octroient le rôle de garde-fou contre "la mauvaise intégration ", l'intégration " à la française " ou " à l'européenne ". Cette intégration stigmatisée serait davantage une acculturation à des comportements occidentaux réprouvés. Le suicide, l'alcoolisme ou encore les conduites sectaires de certains en seraient le fruit. Chez les plus jeunes, cette intégration se traduirait par l'irrespect des parents (fumer, boire de l'alcool, délinquance) et l'impudeur (porter de vêtements trop courts ou moulants pour les femmes, embrasser en public). Elle signifierait la "perte de repères " des jeunes Tamouls contre laquelle le CCT doit lutter en mettant en place des actions de ressourcement culturel et d'engagement politique. Par conséquent, il s'agit de vivre en France en conservant le quant-à-soi sri-lankais et en se consacrant à la " cause tamoule " dans un pays, certes menaçant pour la culture tamoule, mais accueillant pour les exilés sri-lankais. Le projet d'une nouvelle migration ne paraît pas opportun aux yeux des militants nationalistes, a fortiori vers I'ancienne puissance coloniale, jugée en grande partie responsable des maux du Sri Lanka. Leur critique est plus vive encore en 2001 quand I'organisation des LTTE, considérée comme organisation terroriste, y est interdite.

Plus profondément, la condamnation d'une nouvelle migration résulte d'une interprétation nationaliste de l'exil efficiente. Dans la rhétorique séparatiste, la migration sert exclusivement le projet collectif du Tamil Eelam, toute autre considération est proscrite, a fortiori quand elle traduit la quête individuelle d'un meilleur confort de vie. Migrer pour retrouver une position sociale élevée dans la hiérarchie des castes d'avant la guerre ou pour élever sa condition sociale, dans une démarche individualiste, est contraire au récit nationaliste de l'exil et de l'expérience migratoire. Dans ce récit, lesTamouls ont quitté leur terre natale pour fuir la répression cinghalaise, aspirant à y revenir une fois l'indépendance acquise. En conséquence, le temps de l'exil ne peut être qu'un temps de souffrance et de sacrifice, hommage en miroir au " sacrifice suprême " des " martyrs" de la 
guerre, les "Tigres noirs". Vivre loin de la terre natale ne doit pas signifier vivre mieux et y revenir ne peut signifier un retour à la société tamoule antérieure à la guerre et à I'Indépendance. Les LTTE prônent un principe d'égalité et d'unité entre les Tamouls, récusant leurs différences sociales et culturelles internes, fondées en partie sur la hiérarchie des castes, tandis que sont accentuées celles entre Tamouls et Cinghalais, dans une " histoire défensive " (Dequirez, 2011) qui les aurait opposés de tout temps. L'historiographie séparatiste inscrit le combat dans un passé glorieux, antérieur à la colonisation ${ }^{12}$. Les Tigres s'inspirent de l'empire Chola pour leur emblème et le nom de guerre de Prabhakaran résonne avec celui de Karakalan, premier roi Chola. Leur récit mythique s'appuie sur de nombreuses publications d'intellectuels, dont certains sont inféodés aux LTTE ${ }^{13}$. L'ordre militaire est idéalisé, à l'inverse de l'ordre social fondé sur la hiérarchie des castes et de l'âge ${ }^{14}$. L'organisation de la vie des Tamouls dans l'exil doit, à leurs yeux, se rapprocher de cet idéal et être consacrée entièrement à faire vivre le " peuple tamoul ", dont les "communautés tamoules " dans le monde sont des fragments quasi organiques.

\section{Diaspora tamoule versus peuple tamoul}

En effet, les partisans des LTTE défendent une nation ethnicisée basée sur le triptyque : une langue, un peuple, un territoire. Occupant tout l'espace migratoire, du pays d'origine aux pays d'installation, ils distillent leur interprétation de l'exil chez les "expatriés ", des raisons du départ aux expériences migratoires (Fuglerud, 1999). Ils érigent ainsi le " peuple tamoul " en victime de la complaisance, voire de la complicité des Occidentaux avec la présidence cinghalaise. Les militants fustigent le silence, sinon l'indifférence, des médias et politiques Occidentaux à l'égard des "problèmes tamouls", voire leur hostilité en accordant crédit aux accusations du gouvernement sri-lankais à l'encontre des LTTE. Ils déplorent également les obstacles administratifs opposés à la vie associative en France, I'obtention plus difficile du statut de réfugié, I'exploitation des Tamouls dans le travail ou encore leur assimilation méprisante aux "étrangers ", Noirs et Arabes" ${ }^{15}$ (Étiemble, 2002). Cette " histoire alternative "

12 Leurs références sont les trois empires Chola, Pandiya et Chera de I'Inde du Sud (la période Sangam, 300 ans avant et 300 ans après J.-C.) et le royaume tamoul du Nord de I'île, qui perdura jusqu'à I'arrivée des Portugais au XVle siècle.

13 Lors de notre enquête, nous trouvons ces publications en langue française, en vente dans les locaux du CCT, rue des Pyrénées à Paris. Elles sont également accessibles à la bibliothèque de I'INALCO. Pour exemples: Emmanuel S. J. [vicaire général de Jaffna de 1992 à 1997, établi en Allemagne] (1997) Libérez le peuple tamoul !, Suisse, Action des Chrétiens pour l'Abolition de la Torture et Association tamoule du canton de Fribourg ; Irassattiname S. [président de I'association culturelle tamoule du Canada] (1998 [1995]) Un regard sur I'histoire de l'Eelam. Eelam tamoul, le pays et le gouvernement jusqu'en 1977, Paris, Fédération des Associations tamoules ; Julia Jean-Marie [président de la Fédération Tamoule en France] (2000) Le génocide des Tamouls à Sri Lanka, Lyon, Cimade. Inlassablement, ce dernier, bien après la défaite de 2009, continue à écrire et prendre la parole pour dénoncer le sort des Tamouls au Sri Lanka.

14 L'attitude de méfiance à l'égard des parents et des adultes en général est fondatrice de l'idéologie du Mouvement des LTTE, valorisant la jeunesse et rejetant toute hiérarchie due à I'âge et à I'ancienneté. D'ailleurs, le surnom des militants LTTE est The boys en écho au parcours de Prabhakaran ayant créé les Tigres à dix-huit ans et, âgé d'à peine vingt ans, assassiné le maire de Jaffna.

15 Les Tamouls se montrent eux-mêmes très dépréciateurs de ces populations et se sentent humiliés d'y être assimilés. 
(Dequirez, 2011) n'est pas sans effets chez les réfugiés, qui se voient ainsi proposer un sens collectif et partagé de leur existence. Quand nous les entretenons sur leur histoire personnelle, nos interlocuteurs reprennent fréquemment à leur compte l'historiographie séparatiste, énumérant les brimades et tragédies vécues par les Tamouls depuis I'Indépendance. Le "Nous " collectif, souvent victimaire, s'impose dans leurs propos. Les différents médias des séparatistes contribuent à la performativité du récit nationaliste. L'espace Internet reflète singulièrement le " succès de leur rhétorique " (Fuglerud, 1999). En mars 2001, nous répertorions sur le Web mondial, via Google, une quarantaine de sites comprenant les vocables "tamoul ", "tamil " et " eelam ". Les langues utilisées sont I'anglais, le tamoul puis le français. Les sites, localisés au Sri Lanka, en Inde, en Europe, en Amérique du Nord et en Malaisie, s'adressent à un public explicitement " tamoul ", éloigné de sa terre d'origine, dans une palette allant des Tamouls du Sri Lanka ayant fui la guerre aux Tamouls du sous-continent indien ayant émigré à différentes périodes. Informations, divertissements, forums, annonces de toutes sortes forment les rubriques les plus fréquentes dans les sites tamouls liés au Sri Lanka. Au début de la décennie 2000, les LTTE ont également leurs sites officiels - eelam.nation et tamoul.org - construits de manière idoine : des onglets consacrés à I'histoire et aux actualités du conflit, les photographies et déclarations de Prabhakaran, les emblèmes des LTTE et les hommages aux combattants, etc. Par ailleurs, les médias plus traditionnels sont aussi phagocytés par les partisans des LTTE. Deux hebdomadaires de langue tamoule sont édités à Paris dans cette période. L'un d'eux, Eelamurasu ("Tambours de I'Eelam "), émane directement du CCT, le second, Eelanadu ("Pays d'Eelam "), est sous son influence. La direction d'Eelamurasu, né en 1995, nous indique fièrement que le journal est distribué "dans toute l'Europe ", à hauteur de 15000 exemplaires, dont un tiers en France. Le journal traite des événements au Sri Lanka ainsi que de l'actualité française et internationale. Les militants du Mouvement revendiquent leur activité de propagande pour contrer I'hostilité des médias internationaux et cinghalais. Radios et télévisions, dont deux sont situées à Bobigny et à La Courneuve, sont aussi présentes dans le quotidien des exilés tamouls. Les programmes sont ponctués des nouvelles du Sri Lanka et la mise en relation des Tamouls disséminés dans le monde; des informations relatives à la procédure d'asile, au regroupement familial, à la santé, àl'école, etc. Bien que des tensions existent entre des radios et télévisions concurrentes, en raison notamment de leur plus ou moins proximité auxTigres, elles diffusent de la même manière leur historiographie officielle.

Cette diffusion internationale importe avant tout aux sympathisants des LTTE. Ils utilisent toutefois peu encore le terme " diaspora ", lui préférant ceux de "réfugiés", " expatriés " ou " exilés ". Ces termes figurent davantage, il est vrai, la souffrance de la séparation et l'attachement indéfectible au pays tamoul, symbolisé par le combat des LTTE. À l'inverse, le terme "diaspora " entérine une existence en dehors de la terre d'origine, qui devient une référence de plus en plus abstraite. D'ailleurs, les différents canaux d'information du Mouvement s'adressent à la "communauté ", au "peuple ", à la " nation " tamoule en exil. A contrario, nos interlocuteurs davantage distants des LTTE utilisent plus volontiers le vocable " diaspora " évoquant une langue et une culture commune tamoule et indienne. Toutefois, les personnes engagées dans une activité entrepreneuriale à l'échelle internationale, tel le commerce des statuettes pour les temples, circulant au gré des saisons religieuses et des commandes, du Sri 
Lanka à la région parisienne, de Paris à Londres, au Canada ou à La Réunion, nous paraissent s'inscrire davantage dans une "communauté transnationale " (Waldinger, 2006), détachée des frontières et des séparatistes. Quant aux Tamouls sri-lankais réfugiés, leurs sociabilités, leurs références culturelles et leurs échanges entre les pays d'accueil et avec le Sri Lanka les rapprochent d'une " diaspora communautaire " (Bruneau, 2004). Les activités associatives se focalisent sur la transmission de la culture et le lien avec le pays d'origine tandis que les mêmes fêtes et rites religieux rassemblent chaque "communauté " à travers et dans les pays d'installation. Commerces et médias tamouls évoquent au quotidien le Tamil Eelam, tandis que les souvenirs partagés dans les émissions télévisées ou radiophoniques actualisent la " multipolarité " et "l'interpolarité " d'une formation diasporique (Ma Mung, 1995). La réticence du Mouvement des LTTE à l'égard de l'expression " diaspora tamoule " s'inscrit, elle, dans une autre caractéristique de la formation diasporique, conséquence des deux premières. En effet, la " perception d'exterritorialité ", autrement dit " une conscience de soi dans un non-lieu " et "l'impossibilité de la territorialisation " signifie s'identifier à un " non-lieu " (Ma Mung, 1995). L'appartenance à une formation diasporique prime alors sur l'appartenance à un territoire-nation. Les liens de solidarité au sein de la communauté diasporique sont privilégiés, aux dépens des liens avec le pays d'origine. L'expérience diasporique valorise la "culture de la diaspora ", fondée sur les valeurs et les signes de la transnationalité. Des forces contraires alimentent ainsi la dynamique de diaspora, notamment " politique " pour laquelle la référence à la terre-nation est essentielle (Bruneau, 2004) ${ }^{16}$. On comprend, par conséquent, que les militants du Mouvement des LTTE puissent se défier de la duplicité inhérente à la dynamique de diaspora. La " culture de la diaspora ", fondée sur le " pari d'ubiquité " oublieux de la mère-patrie (Ma Mung, 1995), s'oppose à leur lecture nationaliste de l'exil. Pour les séparatistes, seul le Mouvement peut endosser la capacité d'ubiquité sans mettre en péril l'existence même de la nation tamoule. C'est pourquoi, à leurs yeux, les réfugiés tamouls ne peuvent être de France et d'ailleurs, se mouvant dans l'espace diasporique au gré de leurs aspirations, valorisant les signes culturels de l'existence en diaspora au détriment de l'identité du Tamil Eelam. Dans la vision des " amis des LTTE ", si la reconnaissance du statut de réfugié confère une forme de reconnaissance de leur cause en France, les " expatriés " ne peuvent être que de là-bas, duTamil Eelam. À la stabilité du séjour répond une forme de précarité quotidienne, un inconfort matériel et psychologique entretenu par l'emprise idéologique et matérielle des représentants du Mouvement en France. Concrètement, cette emprise se canalise dans la forme associative qui permet aux " militants " d'encadrer le mode de vie des Tamouls réfugiés et leurs enfants.

\section{L'engagement associatif au miroir de la lutte}

Au cours de la décennie 1990, l'activité associative s'est développée et structurée dans les pays d'installation des réfugiés tamouls. Une même organisation, affiliée aux LTTE, le Comité de Coordination des Tamouls (CTT), existe en Angleterre, en Allemagne, en Australie, au Canada, au Danemark, aux États-Unis,

16 Les diasporas peuvent être fondées sur un pôle " entrepreneurial " (les Libanais, par exemple), " religieux " (les Juifs, les Grecs pontiques) ou " politique " (les Palestiniens, les Tibétains). 
en France, en Italie, en Norvège, aux Pays-Bas, en Suède et en Suisse, ainsi que l'indiquent les documents fournis par le Comité parisien. En France, les réfugiés I'appellent aussi communément " l'Association ". Cette désignation, conforme au statut juridique de l'association loi 1901, permet de se fondre dans le tissu associatif ordinaire, sans être associé trop visiblement auxTigres. Le qualificatif "franco-tamoul ", attribué fréquemment aux diverses associations dans les villes d'installation, rejoint cette démarche de discrétion et de légitimité dans la société environnante. En 2000, nous dénombrons une trentaine d'associations établies dans la région parisienne ainsi qu'à Toulouse, Bordeaux, Strasbourg, Lyon ou encore Tours, réunies au sein de la Fédération des associations tamoules de France, elle-même reliée au C.C.T, selon un système d'emboîtement identique à celui des poupées gigognes. Toutes convergent pour s'assurer de la participation des Tamouls à leurs activités et de leur adhésion à la cause.

\section{Soutenir « I'Association "}

Des associations, que nos interlocuteurs qualifient d' $d$ humanitaires " organisent des actions destinées à recueillir des fonds afin de fournir des médicaments, des vivres et des abris aux populations tamoules déplacées dans l'île. L'une des plus actives est l'Organisation pour la Réinsertion des Tamouls (ORT), secondée par Le Comité des Femmes Tamoules ${ }^{17}$, section féminine du CCT, qui gère la logistique des spectacles et des manifestations. De son côté, l'Association des Droits des Tamouls rédige une gazette à l'intention des personnalités politiques des différents États afin de les informer des " atrocités " commises par les Cinghalais envers les Tamouls. Enfin, des bénévoles du CCT préparent le déplacement à Genève lors de la conférence annuelle des Nations Unies sur les Droits de l'Homme afin de faire connaître la tragédie tamoule du Sri Lanka. Les cadres du CCT I'assènent avec force : " tout Tamoul est un Tigre dans I'âme! ". L'ethnicisation de la figure du combattant se traduit pour les réfugiés tamouls par le même devoir, celui de prouver leur identité tamoule en aidant financièrement la lutte. La collecte est au cœur du système associatif mis en place par le Mouvement. D'ailleurs, les membres du CCT et des associations affiliées qualifient avec mépris de "traîtres " et d' "Européens " ceux qui ne le font pas. Ils déplorent que certains, une minorité, une fois "bien installés", se montrent " moins solidaires " et déclinent les sollicitations des émissaires de "I'Association" lors des collectes. Selon nos interlocuteurs, une somme minimale annuelle de 838 euros serait nécessaire pour subvenir aux besoins quotidiens d'une personne au pays et ils se basent sur cette somme pour organiser la collecte auprès des ménages tamouls. Ils modulent les périodes et

\footnotetext{
17 Ce comité a pour objectif premier de recueillir des fonds pour les orphelinats au Sri Lanka. Mais les jeunes militantes, âgées d'une vingtaine d'années, ont aussi pour mission de contrer la " mauvaise intégration " et faire preuve de conduites exemplaires pour les autres femmes et les jeunes. Ainsi, nous dit Dévi, vingt-cinq ans : " $C^{\prime}$ est à nous de montrer comment on doit être. Mais les filles qui grandissent ici, les jeunes, c'est très difficile de leur expliquer comment il faut parler avec les parents, comment il faut parler avec les autres, comment il faut s'habiller. Les filles, ici, prennent trop de libertés. [...] II y a une façon de s'habiller. Le sari par exemple, dans le pays, on le mettait aussi, mais on ne montrait pas notre corps. Si je le mets là-bas, vous ne voyez rien, mais ici ce n'est pas pareil. Pour les Européens, c'est normal. Mais pour nous, ce n'est pas du tout normal." " Quant à I'engagement, les jeunes militantes font l'éloge des Tigresses Noires, unités combattantes féminines bien connues pour leurs attentats suicides. Vidéos à I'appui, les militants du CCT font le récit du sacrifice ultime de celles qui à leurs yeux sont de véritables héroïnes.
} 
les modes de versement selon les ressources des ménages : dons par espèces ou par chèques; versement hebdomadaire, mensuel ou annuel en fonction des sommes et des régions d'habitation. La quête a lieu en général le week-end, de manière plus aisée pour les militants dans la région parisienne et à Paris, qui quadrillent les secteurs résidentiels des Tamouls. Les déplacements en province sont moins fréquents. Les cadres du CCT soulignent l'efficacité et la rationalité de leur système. Des groupes de deux ou trois jeunes "garçons", munis de leur carte du Comité, visitent leurs compatriotes en laissant des "publicités " (tracts) relatant les événements au Sri Lanka et les besoins des populations civiles et des combattants LTTE. Ils précisent la date de leur prochain passage pour la collecte et relèvent alors le nom des donateurs, la somme et les modalités de versement. Aux plus réticents, les jeunes militants décrivent la situation dramatique des Tamouls au pays. Si besoin, d'autres collecteurs, plus aguerris, viendront les voir une nouvelle fois et argumenteront davantage afin qu'ils versent une somme " minimale $"^{18}$. Des campagnes spécifiques sont organisées. L'ORT quête pour "I'intégration au niveau économique ". Le Comité, " c'est pour la lutte ", nous précise-t-on, soit l'achat de matériels nécessaires aux différents corps d'armée : "Tigres Noir ", "Armée de l'Air ", "Armée de Terre ". Les destinations de la collecte évoluent au gré des événements au pays. D'après les dirigeants du CCT, les Tamouls, "traumatisés par les violences cinghalaises ", donnent leur argent sans aucune contrainte, contrairement aux rumeurs lancées par le gouvernement cinghalais afin de discréditer le Mouvement en Occident. Néanmoins, des Tamouls, connaissant souvent des situations financières précaires, nous ont dit redouter le passage des collecteurs du Mouvement. Questionnés à ce sujet, les militants du CCT y voient le signe de la "traîtrise " des intéressés en France ou de leurs proches au pays et affirment ces craintes non fondées au regard de l'absence de plainte en France à leur encontre. Certes, les réfugiés rencontrés affichent plutôt leur sympathie à l'égard des LTTE. Seuls quelquesuns les comparent, à voix basse, aux Khmers rouges ou, plus souvent, à " une mafia ". La plupart ont confirmé leurs dons aux membres du CCT. D'aucuns le disent fièrement, répétant avec conviction le discours militant. D'autres le reconnaissent avec quelque embarras. Enfin, des réfugiés affirment ne rien donner sans être pour autant menacés. À l'aube du XXle siècle, le Mouvement des Tigres suscite globalement une crainte respectueuse, tant il est devenu le symbole unique et exclusif des luttes pour le peuple tamoul au Sri Lanka et dans la diaspora, garant de son identité et de sa culture.

Dans l'exil, les réfugiés tamouls sont sommés de soutenir les combattants du Tamil Eelam et d'adopter un mode de vie proche du leur. L'engagement associatif est à ce titre valorisé. Les associations affiliées au CCT ont toutes pour cœur de cible la " jeunesse tamoule ". Beaucoup, filles et garçons, en sont des militants actifs. Les jeunes militants sont érigés en figure jumelle dans l'exil des jeunes combattants au pays.

\section{La jeunesse en ligne de mire}

Les acteurs associatifs mettent en avant la double nécessité de socialiser les jeunes à " la culture tamoule " et de s'assurer de leur soutien à la " cause tamoule ". Sur ce point, il s'agit de les informer de l'histoire et des événements

18 Le montant minimal ne nous est pas précisé, ni les arguments avancés. 
au Sri Lanka. Les membres du CCT affichent leur défiance à l'égard des parents à ce sujet. Ceux-ci se montreraient peu diserts de peur, nous dit-on, que leurs enfants " deviennent carrément des Tigres et qu'ils s'en aillent là-bas ". Si les parents sont inquiets de l'activisme des représentants du Mouvement, ils sont toutefois séduits par leurs propos sur l'identité et la culture tamoule. En effet, bien que les LTTE affichent une vision égalitaire de la société tamoule, abrasant les hiérarchies de caste, d'âge et de sexe, ils composent aussi avec les traditions en valorisant la vertu féminine, en instaurant la séparation des sexes dans le combat, en jouant du symbolisme maternel dans l'éloge de la mère patrie ou en affirmant la solidarité de castes.

Certes, il existe des associations indépendantes du CCT. Certaines sont d'ordre confessionnel (surtout catholiques), attachées à la paroisse d'origine, et paraissent assez minoritaires dans les années 1990. D'autres associations, dites "littéraires" par leurs adhérents, s'adonnent à la publication de revues et l'organisation de rencontres culturelles d'un pays à l'autre. Leur caractère volontairement élitiste atteint peu les réfugiés tamouls sri-lankais, a fortiori moins scolarisés en raison de la guerre et appartenant à des catégories sociales plus défavorisées. Du reste, ces associations s'adressent à la diaspora tamoule du sous-continent indien, non exclusivement sri-lankaise. La critique politique de I'action des Tigres demeure relativement feutrée chez les acteurs associatifs se déclarant distants, sinon opposés au Mouvement. Les paroles alternatives sont, par conséquent, moins audibles face à la rhétorique des LTTE et de peu de poids face au déploiement associatif de leurs sympathisants. Les activités culturelles " traditionnelles ", les valeurs de respect et de discipline prônées par les acteurs associatifs apaisent les parents quant à leur activisme politique. Les parents sont séduits et rassurés par cette rhétorique, eux qui s'inquiètent de l'effritement de la culture tamoule chez leurs enfants au contact de la société française dont les valeurs et les conduites, notamment celles relatives à la sexualité et au mariage, leur paraissent éloignées des leurs. Dans la décennie 1990, le tissu associatif, structuré par le CCT, devient ainsi aux yeux de nombre de Tamouls sri-lankais le lieu de ressourcement culturel. L'association Tamoulcholaï, créée en 1998, semble particulièrement répondre à cette fonction. Une trentaine d'" écoles Tamoulcholaï " y sont rattachées. Selon son président, Tamoulcholaï signifie le " jardin vert " et la devise de l'école entretient la métaphore : " notre langue maternelle est la racine de notre bien-être ". II s'agit de cultiver les " racines tamoules " des enfants en leur transmettant les valeurs de réussite, de travail et de discipline. Les écoles proposent des cours de langue tamoule, danse et musique traditionnelle, ainsi que du soutien scolaire. Elles accueillent environ 1600 enfants en 2001, âgés de cinq à dix-huit ans. Le spectacle des enfants clôt I'année scolaire, spectacle dans lequel ils chantent, dansent et jouent, habillés en treillis militaires, des scènes épiques glorifiant les " martyrs " de la lutte de libération ${ }^{19}$.

19 En 2018, le réseau des écoles Tamoulcholaï existe toujours malgré le décès de son fondateur. II s'exprime dans les associations franco-tamoules, à travers les cours de langue tamoule qui y sont dispensés selon des programmes, des manuels et des examens établis par le Tamoulcholaï. Si nous ne pouvons actualiser le chiffre de 2001, nous observons toutefois que I'association Tamoulcholaï évoque, sur son site web, I'organisation d'examens à Paris ainsi que dans d'autres villes (Strasbourg, Nice, Rennes, etc.). Par ailleurs, les photographies des galas de fin d'année ne montrent plus d'enfants en uniformes, mais en tenues traditionnelles tamoules. 
L'entretien des " racines tamoules " est un objectif consensuel, entre les familles tamoules de France et les partisans des LTTE et trouve matière à se développer dans la vie associative. Les jeunes sont invités à devenir à leur tour des " militants ". Le terme " militant ", préféré à celui de bénévole, permet d'enchevêtrer les dimensions politiques et culturelles des activités associatives. Nos interlocuteurs l'affirment : le bénévolat fait écho au "sacrifice suprême " des "Tigres Noirs " au Sri Lanka, se battant jusqu'à la mort pour libérer I'Eelam Tamoul. Le militant, confondu alors avec toute la jeunesse tamoule, doit être un " ascète " (Meyer, 2001) entièrement dévoué à la cause. L'engagement associatif signifie la recherche des performances, intellectuelle (réussite scolaire) et physique (réussite sportive), la discipline et le respect des "racines tamoules". Les jeunes sont très présents dans les associations et encouragés à mettre en œuvre leurs propres actions. L'Association des Étudiants tamouls se donne ainsi pour objectif de publier des textes en langue française destinés aux jeunes tamouls scolarisés en France, plus familiers de l'écriture française. Les dirigeants du CCT se félicitent de cette initiative, car elle inciterait les jeunes à devenir à leur tour des " militants". De manière générale, les jeunes sont chargés d'assurer les diverses activités du CCT : tenir ses magasins, travailler au journal, participer à la collecte de fonds, relayer les informations du Mouvement et s'engager dans la vie associative. Des associations développent également des activités sportives ciblant la jeunesse tamoule : football, athlétisme, badminton, volleyball, cricket, etc. Chaque été sont d'ailleurs programmés des tournois entre les différentes associations " à la mémoire des combattants". Les militants du CCT les encouragent comme autant d'occasions d'entretenir chez les jeunes l'esprit de compétition, le désir de se dépasser et de "représenter " fièrement la " nation tamoule".

Des voix dissonantes se font malgré tout entendre au début des années 2000, le rôle du mouvement séparatiste dans la guerre et son emprise sur la diaspora tamoule sont mis en question.

\section{L'héritage associatif dans l'après-guerre}

La défaite donne un coup d'arrêt au système idéologique du Mouvement, toutefois, la dynamique associative en demeure fortement imprégnée.

\section{Une organisation discréditée et la fin de la " cause tamoule " ?}

Le mouvement des LTTE est mis à mal dans les premières années de la décennie 2000, accusé de divers maux, dont l'enrôlement forcé et l'élimination des concurrents au Sri Lanka, ainsi que l'impôt révolutionnaire dans les pays de la diaspora. Désigné en 2006 dans I'Union européenne comme mouvement terroriste, il fait l'objet d'enquêtes financières dans toute l'Europe. Des membres du CCT sont d'ailleurs arrêtés en France en 2007 pour extorsions de fonds liées à des activités terroristes. À l'issue du procès en 2009, la dissolution du CCT est 
prononcé $e^{20}$. Parallèlement au Sri Lanka, les offensives gouvernementales ont raison d'une armée séparatiste affaiblie. La mort du leader des Tigres semble sonner le glas du conflit. Néanmoins, l'expression « fin de la guerre, mais pas la paix " repérée à de nombreuses reprises dans des sites et médias encore actifs laisse à penser que le conflit demeure latent. Par ailleurs, la fin de la guerre se traduit par des déplacements de populations et des exactions de l'armée cinghalaise, dénoncées tant par les ONG que par le gouvernement tamoul transnational, créé en 2010 à New York. Des régions sont encore occupées par l'armée et les forces paramilitaires, bien que la guerre soit officiellement terminée. Des spécialistes du Sri Lanka (Meyer et Mandavan, 2015) préfèrent parler de "période post-guerre " plutôt que de " période postconflictuelle ", malgré la disparation de l'organisation des LTTE. Des décennies de violences et de tensions ont laissé des traces tant au Sri Lanka que dans la diaspora tamoule. Le " nationalisme à longue distance " (Meyer et Mandavan, 2015), orchestré par les militants des LTTE dans les années 1990, est loin d'être éteint. La situation politique reste tendue au Sri Lanka et le quartier de la Chapelle résonne souvent des hommages aux " martyrs " tamouls et des indignations des Tamouls en exil face aux exactions subies par la population tamoule dans le Tamil Eelam. Les portraits de Prabhakaran continuent d'orner les vitrines commerçantes et les commémorations sont constantes. Certes, les sites Internet des LTTE disparaissent après leur défaite ou nombre de pages d'accueil sont désormais inactives. Avant cela, la critique " tamoule " du Mouvement est peu visible dans l'espace virtuel, malgré la multitude de sites gouvernementaux occidentaux (Canada, États-Unis) relatant les exactions des LTTE (terrorisme, trafics) et d'ONG (Amnesty International) dénonçant les atteintes aux droits de I'homme dans les deux camps. La parole publique semble une parole pro-LTTE dans la décennie 1990, tout au moins jusqu'à l'interdiction européenne des LTTE en 2006 et a fortiori la défaite en 2009. Des travaux ultérieurs à 2009 attestent encore de difficultés persistantes à recueillir des " discours dissonants " et la méfiance des Sri-Lankais parisiens à livrer leur opinion (IEDES, 2011). Néanmoins, des voix plus modérées peuvent désormais se faire entendre. La forme diasporique semble, elle, s'épanouir à travers le développement des activités culturelles et religieuses, porteuse de valeurs et de signes associés davantage au partage de liens culturels et linguistiques qu'à celui d'un projet politique (GoreauPonceaud, 2011). Cette dynamique paraît plus visible en France qu'autrefois, se constituant sur une ethnicité tamoule sans substrat nationaliste, voire sans

20 Le 27 octobre 2009, un article de Libération titre : " Le financement de la guérilla tamoule en procès à Paris ". Cet article pose la question : "Racket ou dons volontaires ? Financement du terrorisme ou soutien à la résistance tamoule? ". Une vingtaine de membres présumés des LTTE, sont mis en examen en 2007 pour " association de malfaiteurs en relation avec une entreprise terroriste " et " extorsion de fonds en relation avec une entreprise terroriste ". Les LTTE sont inscrits sur la liste des organisations terroristes de I'UE et donc interdits depuis mai 2006, le parquet estime que le CCT est la "vitrine légale " des LTTE. Il figure parmi les prévenus en tant que personne morale, chargée de cette collecte. En 2006, à la suite d'une "opération spéciale " visant à obtenir 2000 euros par foyer, 7,5 millions d'euros sont réunis. L'enquête du juge Bruguière en 2006 a mis en évidence des actions du groupe "Sathya " chargé d'exercer des " violences physiques " contre les opposants tamouls au LTTE ou de faire pression sur ceux qui ne voulaient pas " participer à la collecte ". Lors du procès, aucune partie civile n'est représentée ni aucun témoin de l'accusation. L'un des hommes impliqués dans ce procès, ancien leader des LTTE et condamné à cinq ans de prison, est assassiné en 2012 en sortant du local du CCT. Resté en détention provisoire jusqu'en juin 2010, sa peine faisait l'objet d'un aménagement. 
terres sinon l'espace diasporique, le monde dans sa globalité, et des hauts lieux de la diaspora tamoule tels que le quartier de la Chapelle. Bien qu'il existe aujourd'hui, d'autres " centralités communautaires " dans la proximité des pôles résidentiels des Sri-Lankais (Lo Duca, 2015), la centralité de la Chapelle demeure évidente pour les Tamouls tant elle symbolise leur présence en France. Le quartier est bien connu desTamouls de la diaspora, même s'ils ne sont jamais venus en France (Dequirez, 2010).

\section{Rhizomes associatifs}

L'interdiction de l'organisation des LTTE, incarnée par le CCT en Europe, affecte grandement les associations inféodées au Mouvement. Pour autant, certaines se sont transformées et d'autres sont apparues, porteuses de projets associatifs qui ne sont pas sans rappeler ceux des " militants" du CCT ; les références à un Tamil Eelam martyrisé y sont fréquentes. C'est le cas de la Maison du Tamil Eelam-France (MTE), créée en 2009, dont le président est alors un ancien membre du CCT. La MTE organise un référendum en décembre 2009 auprès de la population tamoule vivant en France afin qu'elle se prononce sur la création d'un Tamil Eelam séparé. La MTE est également I'un des membres fondateurs du Global Tamil Forum, une organisation internationale regroupant des associations et organisations anciennement pro-LTTE, visant à " promouvoir le droit des Tamouls en tant que peuple et nation, à empêcher la colonisation de la patrie du peuple tamoul par la majorité cinghalaise et à œuvrer pour l'établissement d'un État indépendant et souverain ". La MTE orchestre également les commémorations du " génocide du Juillet Noir " de 1983. Sa page d'accueil reprend les antiennes habituelles sur I'histoire du royaume tamoul avant les colonisations et "I'invasion des Cinghalais " venant de I'Inde du Nord. De même, des comparaisons sont établies entre le "génocide tamoul " et les génocides juifs ou arméniens, photographies à I'appui. Une lettre ouverte au président sri-lankais Rajapaksa le met en demeure de répondre à la question " si tout va bien dans votre pays, pourquoi les Tamouls fuient ? ", en citant les rapports alarmants des ONG. Le site de la MTE récuse également les accusations de terrorisme et de racket du mouvement LTTE en France. II évalue, enfin, la population tamoule vivant en France à 100000 personnes au moins ; chiffre auquel il ajoute la " deuxième génération " née en France : des jeunes qualifiés de " respectueux des lois de la République ", des " enfants tamouls souvent premiers dans leur classe " et "des élèves qui font de hautes études".

Les expressions, les revendications, les dénonciations font écho à la rhétorique séparatiste. Néanmoins, le propos a évolué en adoptant plus volontiers le terme de "diaspora ", doté d'une nouvelle légitimité politique, le nombre des Tamouls dans le monde faisant office de contrepoids au statut minoritaire au Sri Lanka. Les partisans d'un État tamoul indépendant s'adressent désormais aux "Tamouls de France et d'ailleurs". Cette formule se retrouve dans les statuts associatifs déposés par de nombreuses associations et publiés au Journal officiel depuis 2009. Le terme de diaspora paraît plus répandu, exprimant l'adhésion de toute la diaspora tamoule au combat pour l'indépendance, mené désormais dans l'exil. Le 12 novembre 2012, le président de la Maison du Tamil Eelam publie une lettre ouverte dans L'Humanité, dénonçant l'assassinat à Paris le 8 novembre de l'ancien dirigeant du CCT et le soutien complice des médias et des États étrangers à la " lutte génocidaire menée par l'État Sri Lanka contre le 
peuple tamoul ". II évoque plusieurs fois la " diaspora tamoule " en soulignant sa mobilisation constante pour l'indépendance du Tamil Eelam ${ }^{21}$. Quelques temps plus tard, dans un communiqué publié par le site Sortir du colonialisme, il relate, dans un ton très solennel, la cérémonie d'hommage à Nadarajah Mathinthiran, de son nom militant Paruthi ou colonel Regan chez les LTTE, organisée le 24 novembre 2012 dans une grande salle de Saint-Denis ${ }^{22}$. Son propos, repris ci-dessous, met en exergue l'existence d'une diaspora tamoule nombreuse et solidaire, dans cette cérémonie qu'il décrit longue de plusieurs heures en raison de l'affluence.

"Venus de toutes les régions de France et toute l'Europe en autocars ou en train

(Grande-Bretagne, Norvège, Suisse, Allemagne, etc.) des groupes de la diaspora tamoule ont rejoint Paris. La salle de 3000 sièges était comble, ses allées et travées étaient remplies de monde, et une foule conséquente attendait calmement à l'extérieur tandis qu'une lente procession ne cessait de circuler entre le dehors et la salle. Plus de 10000 personnes ont défilé entre 10 heures du matin et 16 heures. L'endroit était rempli de fleurs. Les drapeaux de pays accueillant une importante diaspora tamoule (France, Grande Bretagne, etc.) avoisinaient ceux du Tamil Eelam. La cérémonie commença par le lever des couleurs : devant la salle comble debout, le drapeau du Tamil Eelam fut porté en haut d'un mat, puis installé en berne. Puis, un défilé de jeunes tamouls avec fanfare funèbre précédait le cortège accompagnant le cercueil de M. Nadarajah MathinthiranParuthi, lui-même suivi d'une procession de représentants d'associations tamoules, d'élus de la République française, et d'amis européens. Commencée à 10 heures du matin, la cérémonie a pris fin à 16 heures. [...] L'immense diaspora tamoule répartie dans le monde est en grande partie constituée de personnes épuisées par les persécutions et ayant cherché refuge ailleurs. Partout, les Tamouls sont appréciés pour leur gentillesse, leur productivité au travail, leur sens de l'organisation, et leur calme. Les responsables de la compagnie de C.R.S. (Police nationale de la République française) déployée à SaintDenis le 24.11.12 commentaient d'ailleurs "être impressionnés par une aussi grande foule tellement calme". Pour nombre des exilés, le rêve est celui d'un retour sur la terre ancestrale, le Tamil Eelam, une fois que celui-ci sera une terre de liberté et de démocratie. Et comme tous les Juifs des diasporas ou les Palestiniens se saluaient partout dans le monde en ces termes pendant les siècles : "L'année prochaine à Jérusalem", les Tamouls de la diaspora rêvent en ces termes: "L'année prochaine à Jaffna"! " (Communiqué du président de la Maison du Tamil Eelam ${ }^{23}$ )

Dans son hommage, le président de la MTE souligne les qualités de la " diaspora tamoule " et selon lui, son rêve toujours vif de retour auTamil Eelam, à l'instar de la diaspora juive, transformant l'expression "L'année prochaine à Jérusalem " en "L'année prochaine à Jaffna ! ".

La MTE édite également une revue sur son portail intitulée Liberté-Le mensuel des diasporas tamoules francophones. Dans les numéros de 2013, un article

21 Cf. URL : https://www.humanite.fr/tamouls-sri-lanka/lassassinat-dun-militanttamoul-paris-le-silence-des-medias-508617

22 Le quotidien Le Parisien fait écho de cet événement très suivi par les Tamouls. Cf. URL : http://www.leparisien.fr/espace-premium/paris-75/plusieurs-centaines-de-tamoulspleurent-leur-leader-assassine-27-11-2012-2357761.php

23 Extrait du communiqué de Thiruchchoti T. [président de la Maison du Tamil Eelam] (2012) Un hommage et une cérémonie silencieuse pour la Liberté du peuple tamoul du Sri Lanka, [en ligne]. URL : http://anticolonial.net/spip.php?article2651 
compare la " résistance tamoule " avec la " résistance française " de la Seconde Guerre mondiale et la qualifiant de "lutte de libération contre une puissance discriminatrice ". C'est là un procédé ancien de la rhétorique des Tigres de convoquer des icônes de la résistance politique, mises au ban de la société avant d'être célébrées pour leur lutte. Les séparatistes dressent ainsi un panthéon du combat politique contre l'oppression où figure désormais Prabhakaran aux côtés de De Gaulle et Mandela. Les actualités sont toutefois datées sur le site de la MTE. C'est le cas pareillement des associations solidaires du Mouvement telles que I'ORT, toujours sise dans le quartier de la Chapelle. Elle décline trois priorités au Sri Lanka : la construction de logements, celle des écoles et des orphelinats et la formation professionnelle des jeunes. En France, elle développe deux activités : d'une part, " sensibiliser les Français à la souffrance desTamouls et leur proposer notre structure comme terrain d'action pour leurs projets ", d'autre part, " faire découvrir nos arts et traditions et favoriser les liens d'amitiés français et tamouls ". Elle organise donc des "soirées tamoules " dans différentes communes et chaque année "la Grande Rencontre sportive tamoule ", basée sur la découverte de jeux et sports traditionnels du Sri Lanka. En 2013, le site Tamil Express fait cependant écho des grandes difficultés financières de I'ORT, lourdement endettée, déplorant la moindre mobilisation des Tamouls lors des événements organisés par l'ORT et le retrait des subventions par les collectivités locales. Une recherche sur Internet en novembre 2017, selon les mêmes modalités qu'une dizaine d'années auparavant, débouche souvent sur des sites ou des pages Facebook inactifs ou fermés. Pour autant, si les associations et les organes de communication " historiques " des séparatistes semblent avoir disparu, la politisation des réfugiés tamouls reste vive dans la dénonciation de I'action gouvernementale au Sri Lanka à l'encontre des Tamouls.

\section{Conclusion : " âge d'or " de l'emprise nationaliste et dynamique associative " totalisante "}

Aux débuts des années 2000, notre objectif initial était de rendre compte des migrations des Tamouls de France vers I'Angleterre. Nous avons dû rapidement réorienter notre étude tant ces migrations restaient à l'état de projet ou bien les intéressés disparaissaient pour ne plus être dans "l'ombre desTigres " et se voir accusés de traitres à la cause en ayant entrepris une migration discréditée par les séparatistes. En effet, pour les militants nationalistes, la migration ne peut être alors qu'à " sens unique " : il s'agit de se consacrer à " la cause tamoule " en attendant le retour dans le Tamil Eelam. Ainsi, nous avons cherché à mieux comprendre l'emprise des nationalistes sur les réfugiés tamouls, emprise qui pouvait entraver des velléités de mobilités migratoires. C'est ici qu'est apparu le rôle clé de la structuration associative, pyramidale, dans la diffusion de I'idéologie nationaliste. La dynamique associative entremêle le politique et le culturel, et constitue à ce titre une force de frappe importante des LTTE auprès des réfugiés tamouls et des jeunes générations, quand bien même certains se montrent réticents à leur égard. Aux débuts des années 2000, notre terrain reflète une période où l'emprise des militants séparatistes est très palpable dans le quotidien des Tamouls sri-lankais et à la Chapelle, quasi-quartier général du Mouvement en France, derrière les devantures commerciales ou religieuses anodines. Cette période constitue, nous semble-t-il, l'« âge d'or " pour l'organisation des militants nationalistes dans son influence sur les Tamouls en France. 
Une dizaine d'années plus tard, après l'interdiction des LTTE en Europe en 2006 et la fin du conflit en 2009, on peut supposer que cette influence est moins prégnante. Interroger de nouveau les projets migratoires des Tamouls installés en France vers l'Angleterre ou le Canada pourrait nous éclairer sur le relâchement de l'emprise nationaliste. Une enquête de terrain serait nécessaire pour y répondre. Quant à la dynamique associative repérée aux débuts des années 2000, des indices témoignent de sa pérennité, notamment dans sa dimension " englobante ". En effet, dans cette période, le projet des sympathisants des LTTE est d'occuper le quotidien des réfugiés tamouls, jeunes et moins jeunes afin d'entretenir leur adhésion à la " cause tamoule " (la lutte pour l'indépendance et la défense du peuple tamoul). Sur ce plan, I'héritage militant est visible dans la dynamique associative, car le caractère " totalisant " se manifeste dans la polyvalence des activités déclarées dans les statuts associatifs. En effet, de nouvelles associations " tamoules " sont régulièrement publiées au Journal Officiel. Les projets, définis dans les statuts associatifs, s'énoncent dans des termes proches, voire identiques, d'une association à l'autre, qu'elle se situe à Strasbourg, Toulouse ou Sarcelles ${ }^{24}$. Ces projets sont relativement " polyvalents " au regard des objectifs annoncés. Ils comportent, dans un ordre dispersé, plusieurs rubriques parmi les suivantes : "créer des cours de soutien scolaire français ; aider les familles en difficulté à trouver un emploi ; organiser des loisirs et le développement culturel ; aider à l'apprentissage de la langue tamoule, française et anglaise, proposer diverses activités manuelles (couture, poterie, etc.), animation et spectacle (danse, théâtre, comédie musicale), sortie, découverte (musée, château, etc.) ; aider à l'intégration et à la compréhension des courriers administratifs, accompagner les personnes aux rendez-vous administratifs, hôpitaux; motiver les jeunes enfants et les adultes à pratiquer une activité physique et organiser des compétitions sportives nationale, européenne et internationale, etc. ". Cet inventaire hétéroclite, non exhaustif, nous semble illustrer une même volonté des acteurs associatifs d'embrasser différents aspects de la vie quotidienne des "Tamouls de France et d'ailleurs ". En outre, la place faite au sport dans les associations est remarquable alors que ces associations ne se déclarent pas comme des associations sportives, excepté la "Fédération nationale des comités sportifs tamouls ", créée en 2013. Le mimétisme avec le sport fédéral est patent, et plus globalement avec la forme associative française, tout en s'en écartant par la polyvalence quasi systématique des activités. La polyvalence des activités associatives symbolise l'héritage politique du Mouvement des LTTE chez les Tamouls sri-lankais et leurs enfants. Cet héritage n'est pas sans lien avec la situation difficile des Tamouls au Sri Lanka dix ans après la fin du conflit. Cet héritage peut-il maintenir les Tamouls à distance de la société française, comme le faisaient les représentants des LTTE, ou les amener à définir de nouvelles modalités d'intégration ? Observer le devenir de cet héritage ne manquera pas, nous l'espérons, de susciter l'intérêt des chercheurs afin de mieux connaître une population marquée par l'ombre

24 Nous avons consulté le site du Journal Officiel avec l'occurrence " tamoul " en ne comptabilisant pas celles qui ont un projet plus religieux, souvent situées à la Réunion. Ce relevé, sur 2006-2016, montre le dynamisme associatif et la récurrence des projets associatifs "tamouls ". Une recherche sémantique mériterait un plus long développement. Cf. URL : http://www.journal-officiel.gouv.fr/association/index.php?ctx=eJyLz2FIK2 KIL8tjSCwuzk9miC9kKEnMzS*NYYjPyCxxK83JYTAyZQAA8HgMAA_\&page=3\&WHAT=ta moul 
des Tigres, toujours peu visible dans notre champ de vision, quasi " exotique " par rapport à des immigrations historiquement plus familières. C'est pourtant, rappelons-le, la première population réfugiée statutaire en France et la connaissance du devenir des Tamouls du Sri Lanka, après le statut, est à ses prémisses. L'évolution de la vie associative nous semble être un prisme pertinent pour comprendre ce devenir.

\section{Références bibliographiques}

Bruneau Michel (2004) Diasporas et espaces transnationaux, Paris, Economica, $249 \mathrm{p}$.

Costa-Lascoux Jacqueline et Du Cheyron Patrick (2004) Quand la recherche française investit les circulations migratoires, Revue Française des Affaires sociales, 2, pp. 183-205.

Dequirez Gaëlle (2011) L'histoire de Sri Lanka vue par les associations nationalistes tamoules en France, Hommes et Migrations, 1291, pp. 72-81.

Dequirez Gaëlle (2010) Processus d'appropriation et luttes de représentation dans le "Little Jaffna " parisien, Revue Européenne des Migrations Internationales, $26(2)$, pp. 95-116.

D’Halluin Estelle (2015) La quête d'asile des exilés sud-asiatiques en France, Migrations Société, 161, pp. 173-186.

Étiemble Angélina (2004) Les Tamouls du Sri Lanka dans la région parisienne. L'emprise du politique, Revue Française des Affaires sociales, 2, pp. 145-164.

Étiemble Angélina (2002) Les Tamouls du Sri Lanka en France. Les arcanes de la "bonne éducation ", Ville-École-Intégration Enjeux, 131, pp. 176-190.

Étiemble Angélina (2001) Les ressorts de la diaspora tamoule. Associations, médias et politique, Rapport ADERIEM/MIRE, $160 \mathrm{p}$.

Étiemble Angélina (2000) Les Sri Lankais dans la région Île-de-France. De l'accueil à l'installation : le rôle du communautaire, Rapport FAS/ADERIEM, 157 p.

Fuglerud Øivind (1999) Life on the Outside. The Tamil Diaspora and Long Distance, London, Pluto Press, 203 p.

Gazagne Philippe (2011) Les Tamouls de La Chapelle. Entre solidarité et dépendance, Hommes et Migrations, 1291, pp. 82-93.

Goreau-Ponceaud Anthony (2013). La Chapelle : un lieu de mémoire pour la diaspora tamoule ?, Conserveries mémorielles, 13, [en ligne], consulté le 11/11/2017. URL : https://journals.openedition.org/cm/1341

Goreau-Ponceaud Anthony (2012) Expériences de racinements au sein de la migration tamoule sri-lankaise, Hommes et Migrations, 1297, pp. 56-66.

Goreau-Ponceaud Anthony (2011) L'immigration sri lankaise en France. Trajectoires, contours et perspectives, Hommes et Migrations, 1291, pp. 26-39.

Haut Commissariat des Nations unies pour les réfugiés (HCR) (2000) Les réfugiés dans le monde. Cinquante d'action humanitaire, Paris, Editions Autrement, $337 \mathrm{p}$. 
Institut d'Étude du Développement Économique et Social (IEDES) (2011) La population tamoule à Paris. Etat des lieux de la recherche et témoignages associatifs, Note de synthèse, réfugiés et asile, $17 \mathrm{p}$.

Lebon André (2000) Immigration et présence étrangère en France 1997/1998, Paris, La documentation française, $129 \mathrm{p}$.

Legoux Luc (1995) La crise de l'asile politique en France, Paris, Les études du CEPED, $262 \mathrm{p}$.

Lo Duca Raphaël (2015) La diaspora tamoule : de la migration internationale à l'ancrage commercial en Île-de-France, [en ligne]. URL : http://www.revueurbanites.fr/chroniques-la-diaspora-tamoule-de-la-migration-internationale-alancrage-commercial-en-ile-de-france/

Ma Mung Emmanuel (1995) Non-lieu et utopie : la diaspora chinoise et le territoire, in Michel Bruneau Éd., Les diasporas, Paris, Reclus, pp. 163-173.

Meyer Éric (2001) Sri Lanka. Entre particularismes et mondialisation, Paris, La Découverte, $184 \mathrm{p}$.

Meyer Éric et Mandavan Delon (2015) Sri Lanka : les séquelles de la guerre, Hérodote, 3 (158), pp. 219-237.

Pétonnet Colette (1982) L'Observation flottante. L'exemple d'un cimetière parisien, L'Homme, 22 (4), pp. 37-47.

Portes Alejandro (1999) La mondialisation par le bas. L'émergence des communautés transnationales, Actes de la Recherche en Sciences Sociales, 129, pp. 15-25.

Robuchon Gérard (1995) Tamouls Sri-lankais en France, Rapport DPM, 173 p.

Waldinger Roger (2006) "Transnationalisme " des immigrants et présence du passé, Revue Européenne des Migrations Internationales, 22 (2), pp. 23-41. 


\section{Angélina Étiemble}

Tamouls du Sri Lanka en France : politisation de l'exil dans l'ombre des Tigres

Le conflit armé au Sri Lanka, opposant la majorité cinghalaise et la minorité tamoule, prend fin en 2009 avec la défaite des LTTE (Libération Tigers of Tamil Eelam). Tout au long du conflit, des Tamouls ont cherché refuge en France et y ont obtenu asile, devenant la première population réfugiée dès la fin de la décennie 1990. Réfugiés statutaires, certains souhaiteraient néanmoins partir en Angleterre pour des raisons socio-économiques. Mais ces projets se heurtent à l'interprétation nationaliste de la migration. Selon les LTTE, la migration est toute entière consacrée au soutien de la " cause tamoule ". Leurs sympathisants sont très influents auprès des réfugiés tamouls de la région parisienne aux débuts des années 2000 via les associations qui leur sont affiliées. Ces associations mêlent habilement les dimensions politiques et culturelles afin d'assurer l'adhésion desTamouls à leur cause. Si cette période représente un " âge d'or " de l'influence des militants nationalistes, avant l'interdiction de leur organisation en Europe en 2006 et la défaite en 2009, nous en percevons quelques échos dans les associations " tamoules" de la décennie suivante.

\section{Tamil of Sri Lanka in France: \\ Politization of the Exile in the Shade of the Tigers}

The armed conflict in Sri Lanka, opposing the Singhalese majority and the Tamil minority, ends in 2009 with the defeat of the LTTE (Release Tigers of Tamil Eelam). Throughout the conflict, the Tamil ones sought refuge in France and obtained asylum there, becoming the first population taken refuge as of the end of the decade 1990. Statutory refugees, some would wish nevertheless to leave for England for socio-economic reasons. But these projects run up against the nationalist interpretation of the migration. According to the LTTE, the migration very whole is devoted to the support of the "Tamil cause".Their sympathizers are very influential to the Tamil refugees of the Paris region at the beginning of the years 2000 via associations which are affiliated for them. These associations skilfully mix political dimensions and cultural in order to ensure the adhesion of Tamil their cause. If this period represents a "golden age" of the influence of the nationalist militants, before the prohibition of their organization in Europe in 2006 and the defeat in 2009, we perceive of them some echoes in "Tamil" associations of the following decade.

\section{Tamiles del Sri Lanka en Francia: politización del exilio en la sombra de los Tigres}

El conflicto armado en Sri Lanka, que opone la mayoría cingalesa a la minoría tamil, se acabó en 2009 con la derrota de los LTTE (Tigres de Liberación de laTierraTamil Eelam). A lo largo del conflicto, unos Tamiles buscaron refugio en Francia y obtuvieron allí asilo, siendo la primera población refugiada desde el final de la década 1990. Aunque sean refugiados estatutarios, a algunos sin embargo, les gustarían irse a Inglaterra por razones socio-económicas. Pero estos proyectos se enfrentan a la interpretación nacionalista de la migración. Según los LTTE, la migración se dedicó exclusivamente a dar apoyo a la "causa tamil». Sus simpatizantes tienen mucha influencia hacia los refugiados tamiles de la región parisina desde los principios de los años 2000 gracias a las asociaciones a las que se afilian. Esas asociaciones manejan con habilidad las dimensiones políticas y culturales para asegurar la adhesión de losTamiles a su causa. Si ese período representa la "edad de oro" de la influencia de los militantes nacionalistas, antes de la prohibición de su organización en Europa en 2006 y la derrota en 2009, ya percibimos algunos ecos en las asociaciones tamiles de la década siguiente. 